\title{
Orthonome - a new pipeline for predicting high quality orthologue gene sets applicable to complete and draft genomes
}

(1) CrossMark

\author{
Rahul V. Rane ${ }^{1,2^{*}}$ D, John G. Oakeshott ${ }^{2}$, Thu Nguyen ${ }^{1}$, Ary A. Hoffmann ${ }^{1}$ and Siu F. Lee 2,3
}

\begin{abstract}
Background: Distinguishing orthologous and paralogous relationships between genes across multiple species is essential for comparative genomic analyses. Various computational approaches have been developed to resolve these evolutionary relationships, but strong trade-offs between precision and recall of orthologue prediction remains an ongoing challenge.
\end{abstract}

Results: Here we present Orthonome, an orthologue prediction pipeline, designed to reduce the trade-off between orthologue capture rates (recall) and accuracy of multi-species orthologue prediction. The pipeline compares sequence domains and then forms sequence-similar clusters before using phylogenetic comparisons to identify inparalogues. It then corrects sequence similarity metrics for fragment and gene length bias using a novel scoring metric capturing relationships between full length as well as fragmented genes. The remaining genes are then brought together for the identification of orthologues within a phylogenetic framework. The orthologue predictions are further calibrated along with inparalogues and gene births, using synteny, to identify novel orthologous relationships. We use 12 high quality Drosophila genomes to show that, compared to other orthologue prediction pipelines, Orthonome provides orthogroups with minimal error but high recall. Furthermore, Orthonome is resilient to suboptimal assembly/annotation quality, with the inclusion of draft genomes from eight additional Drosophila species still providing >6500 1:1 orthologues across all twenty species while retaining a better combination of accuracy and recall than other pipelines. Orthonome is implemented as a searchable database and query tool along with multiple-sequence alignment browsers for all sets of orthologues. The underlying documentation and database are accessible at http://www.orthonome.com.

Conclusion: We demonstrate that Orthonome provides a superior combination of orthologue capture rates and accuracy on complete and draft drosophilid genomes when tested alongside previously published pipelines. The study also highlights a greater degree of evolutionary conservation across drosophilid species than earlier thought.

Keywords: Orthologue, Inparalogue, Gene duplication, Gene birth

\section{Background}

Distinguishing orthologous and paralogous relationships among gene complements of whole genomes in different eukaryote species is crucial to understanding patterns of functional conservation and change through the course of evolution [1]. Analyses of these patterns could become far more powerful with the rapid growth in the

\footnotetext{
* Correspondence: rahul.rane@csiro.au

${ }^{1}$ Bio21 Institute, School of Biosciences, The University of Melbourne, Melbourne, Victoria, Australia

${ }^{2}$ CSIRO, Canberra, Australian Capital Territory, Australia

Full list of author information is available at the end of the article
}

number of species for which whole genome sequences are available.

Several methods have been developed over the past two decades for orthologue prediction, including tree-based methods (Ensembl Compara [2], PANTHER [3] and PhylomeDB [4]), graph-based methods (i.e., based on pairwise comparisons; Best Reciprocal Hits [5], Reciprocal Smallest Distance (RSD), EggNOG [6], Hieranoid [7], InParanoid [8], OMA [9] and OrthoInspector [10]) and meta-methods combining phylogenetic information derived from different databases (e.g. MetaPhors [11]). Most of these methods were developed prior to the widespread 
availability of whole genome data so they are not well suited to exploit the contextual information (e.g. positional information or splice variation) provided by such data [12]. Additionally, the graph-based methods involve finding genes in pairs of genomes that share the strongest sequence similarity or have the least genetic distance. However, phenomena such as violations of molecular clock behaviour or rapid gene duplications can compromise their performance $[1,13]$. Tree-based methods, on the other hand, typically improve the quality of orthologue inference in the presence of such phenomena [13] and further improvements can be made if synteny information is also used (e.g. BBH-LS [14] or the recently developed MSOAR [15-17]). The MSOAR algorithms utilise genomic context in a genome evolution framework to predict orthologues without relying exclusively on either gene homology or conserved gene order $[12,16,17]$. However MSOAR is unsuitable for analysing draft genomes because it requires chromosome-level assignment of annotated gene models. It also uses approximate measures of genetic similarity rather than the more accurate Smith-Waterman alignments.

The Orthonome orthologue prediction pipeline presented here overcomes the issues highlighted above. Orthonome uses whole genome data but is tolerant of fragmented draft genomes. The pipeline also has a toolkit of scripts to enable orthologue-based whole genome phylogenetic analyses. Orthonome delivers high quality orthologue predictions among eukaryote genomes while maintaining high recall compared to other pipelines. However, Orthonome in its current implementation may not be suited to prokaryote genomes where the prevalence of horizontal gene transfers can confound the evolutionary relationships across species [18]. The accuracy and sensitivity of the pipeline are validated using tests developed by Altenhoff, et al. [19] and published data for twenty Drosophila genomes, twelve of which have been extensively curated.

\section{Implementation}

The Orthonome pipeline

Orthonome is a multimodular pipeline and toolkit for orthologue prediction and phylogenetic and gene evolution analysis. The workflow consists of five major steps as summarised in Fig. 1 and detailed as follows:

\section{Step 1: Processing input files}

Orthonome uses whole genome annotations in GFF3 format and genomes in FASTA format to predict orthology. The processing script 'Gff2gene_zeroidx.py' extracts nucleotide coding sequences and their respective translations. This step is tolerant of annotations with gene models split across more than one contig/ scaffold, making it applicable to splice-alignment-based gene prediction from fragmented draft assemblies such as those produced by Scipio [1] and ExonMatchSolverpipeline [2]. Orthonome retains the longest isoform if multiple splice variants are predicted.

Sequence similarity metrics for genes in each species pair are then calculated using BLASTP [3] and SmithWaterman alignments for each within and between species combination.

\section{Step 2: Pair-wise species comparisons and inparalogue identification}

Following MSOAR [16], for each pair of species, the BLASTP results from Step 1 are processed to create clusters of genes with similar domains using MCL clustering [20]. Both syntenic and non-syntenic inparalogues are then identified within each cluster using neighbour-joining trees based on DNA distance matrices [21] built using locally optimised codon alignments (MAFFT (L-INS-i) [22] followed by PAL2NAL (v14) [23]). The genes identified as inparalogues are not utilised for further analysis in Step 3, thus retaining only the genes classified as ancestral in origin. This step also serves as a filtering step for genes violating expected phylogenetic relationships, such as horizontal gene transfers (HGT) [18] since they will very likely be identified as spurious inparalogues or de novo genes at this stage, depending upon the evolutionary origin of the HGT event in the species analysed. 
Step 3: Score adjustment for pairwise orthologue prediction For each gene retained in Step 2, the five genes with highest sequence similarity are identified from the species compared, based on the fragment bias-corrected pairwise gene similarity score, $S^{\prime}$. These gene pairs are also required to have a low gap extension penalty $(-1)$, Smith-Waterman alignments spanning $>50 \%$ of matched gene lengths with BLASTP bit scores $\geq 30$, and e-values $\leq 10 \mathrm{e}-7$. The reduced gap penalty helps accommodate large gaps between coding regions, allowing more efficient recovery of orthologues that have fragmented gene models (Additional file 1: Note 1) or are significantly diverged. The $S^{\prime}$ scores for each gene pair $\left(G_{a 1}\right.$ and $\left.G_{a 2}\right)$ are calculated using a Smith Waterman alignment and BLOSUM50 (BL50) substitution matrix as follows:

$S^{\prime}=\left(\frac{\text { SmithWaterman }^{B L 50}\left[G_{a 1} \text { vs } G_{a 2}\right]}{\text { Pairwise alignment length }(b p)}\right) *$ percent pairwise identity.

In the estimation of the $S^{\prime}$ score, the pairwise identity calculated from the alignment is multiplied by 100 to create a percentage metric similar to those used by most other orthologue identification pipelines. The number of most similar genes analysed is restricted to five for the purposes of computing speed, which is unlikely to affect orthologue discovery since most tandem inparalogues have already been removed in Step 2.

\section{Step 4: Orthologue prediction and re-calibration to include positional orthologues}

Orthonome uses the modified Smith-Waterman scores of the genes and their corresponding genomic coordinates as inputs for the MSOAR algorithm [17] to identify the orthologous relationships between genes of each species pair. Due to the more sensitive identification of orthogonal relationships using the $S^{\prime}$ score, phylogenetically close neighbours including positional inparalogues are re-analysed to re-calibrate orthologue calls after an initial MSOAR run using synteny information from the genome to fill gaps [16] and reassign true orthologues using micro-synteny (spanning windows of six genes). The $S^{\prime}$ scores and syntenic relationships therefore allow Orthonome to extend the MSOAR analysis and recover orthologues misidentified as inparalogues.

The pairwise orthologues are then used to create clusters of genes using the MCL algorithm with an inflation parameter of 2 [20]. FastTree2 is then applied to orthogonal clusters with only one gene from each species, to construct a consensus phylogenetic tree topology based on randomly concatenated codon alignments.

The gene families constructed using an all-by-all matrix of sequence similarity are then reconciled into orthologous clusters by MultiMSOAR2.0 [17]. Tree reconciliation and formation of orthogonal sets of orthologues further utilises the $\mathrm{S}^{\prime}$ score to quantify sequence similarity between genes. These orthogonal sets of orthologues are termed orthogroups (OG's).

\section{Step 5: Orthogroup clustering and evolutionary classification}

Orthonome partitions gene sets into four orthogonal classes, namely orthologues that are present in all species (hereafter termed 1:1 (n=all) orthogroups), those present in some but not all species (1:1 (1<n<all) orthogroups), inparalogues and de novo gene births (i.e. no significant sequence homology to any other genes in the data set). The orthogroups and inparalogues are further clustered by domain similarity using protein sequence match followed by repeated MCL clustering with zero edge weights for duplications and de novo genes to form super orthologue groups (SOGs). These are akin to functional gene families and consist of orthologues (satisfying at least two of the bi-directional sequence similarity, phylogenetic and syntenic sources of evidence), inparalogues and gene births. Each SOG is named using a four letter alphabet combination code (e.g. ABCD) and orthogroups in the SOG are further numbered as suffixed to the SOG ID (e.g. ABCD0001, ABCD-0002), while the inparalogues and de novo genes are classified with the DUP (inparalogue) and DNV (de novo) codes respectively.

The Orthonome pipeline produces tabulated, databasetool-friendly formats which are utilised to build the web interface and an online database (http://www.orthonome.com). The current implementation includes output data from the 20 Drosophila species analysed in this study.

\section{Computational requirements}

The Orthonome pipeline consists of UNIX bash and c++ scripts assisted by toolkits written in Perl and python. Furthermore the pipeline is written in a map-reduce protocol leveraging the parallelisation enabled by a GNU parallel utility [24]. The source code is available at https://bitbucket.org/rahulvrane/orthonome while the database website is hosted at www.orthonome.com.

The pipeline has been tested on Linux servers with four cores and 64 GB memory hosted on the NeCTAR research cloud [25] and requires peak memory usage of 8 GB. The novel map-reduce implementation in Orthonome reduces the time required by the BLASTP search, Smith-Waterman alignment scoring and orthology assessment by $5-10$ fold compared to a when programs are run with internal multi-threading, albeit with phylogenetically more distant species still taking longer to compare than more closely related species. The mapreduce based programming models often involve implementing calculations and computational tasks in a parallel distributed manner. The approach to pipeline creation allows optimal usage of computing resources 
over a computing grid or local computers and is therefore highly scalable from the 20 genomes tested here to hundreds of genomes. An optimised pre-processing step for generation of sequence similarity scores and a 64-bit recompilation of the core algorithms permit completion of pairwise comparative analyses up to three times faster than the original MSOAR2 implementation.

\section{Web interface}

A complementary web interface is currently available at www.orthonome.com. The web interface allows users to search the database using gene identifiers, gene names or protein/nucleotide sequences in order to view the entire orthogroup and super orthogroup (including genes tagged as inparalogues and closely associated orthogroups clustered by domain) to which the gene in question belongs. Additionally, for each gene analysed in the current implementation, users can also view the summary protein domains [26] and orthologue allocation and interactively view and analyse alignments of the gene with all its orthologues. All the orthologue data and alignments can be downloaded in XLSX, CSV, TXT, FASTA and OrthoXML formats. The output can be directly exported into other data manipulation software.

\section{Comparisons between Orthonome and other pipelines Data used in the comparisons}

The genome and annotation data for the implementation of Orthonome herein use the 12 Flybase Drosophila species (Drosophila ananassae, D. erecta, D. grimshawi, D. melanogaster, D. mojavensis, D. persimilis, D. pseudoobscura, D. sechellia, D. simulans, D. virilis, D. willistoni, D. yakuba; accessible at ftp://ftp.flybase.net/genomes/) [27] and the additional eight draft genomes in modENCODE (D. biarmipes, D. bipectinata, D. elegans, D. eugracilis, D. ficusphila, D. kikkawai, D. rhopaloa, D. takahashii; https://www.hgsc.bcm.edu/arthropods/drosophila-modencode-project) (Additional file 1: Table S1). A total of 308,667 genes were analysed using this approach across the 20 species (Additional file 1: Table S1). We did not use the 66 metazoan species set proposed by Altenhoff, et al. [19] because Orthonome depends upon whole genome information, including both genome sequence and annotation, which is unavailable for most of the 66 species.

The three performance comparisons were carried out as follows:

1. Comparison between Orthonome and five other orthologue prediction pipelines.

The six orthologue prediction pipelines (Orthonome, OrthoDB [28], Reciprocal bi-directional hit, MetaPhors
[11], OMA (two modes: groups and GETHOGS) [9] and MultiMSOAR2 [17]) were compared according to methods which Altenhoff, et al. [19] developed to (i) evaluate phylogenetic discordance between species trees and gene trees constructed using orthologue sets, and (ii) measure the number of orthologue clusters with representatives from all species - representing a recall of conserved orthologues. For pipelines that do not produce orthogonal 1:1 orthogroup predictions (e.g. RBH, OrthoDB and GETHOG's), we used the Quest for Orthologs method (as proposed by the authors) to sample a maximal path across all species, starting with a randomly selected gene and selecting a gene from the 'next' species and resolving soft polytomies (or one:many relationships) using random selection and bootstrapping (see Altenhoff, et al. [19]). We did not use the gene tree or ontology-based methods of comparison from Altenhoff, et al. [19] because only one of the 20 species has the manually annotated gene ontologies and gene trees required for those tests.

2. Comparison between Orthonome and the published data on the 12 drosophila genomes obtained from Flybase.

The output statistics from Orthonome were compared to those generated through the OrthoDB pipeline from Flybase (OrthoDB v7) (https://flybase.org/static_pages/downloads/FB2015_04/genes/gene_orthologs_fb_2015_04.tsv.gz using the script Orthonome_discordance_data.py (see Additional file 1). All orthogroups unique to Orthonome were tested for gene ontology enrichment using the goatools pipeline (https://github.com/tanghaibao/goatools) and enrichment was carried out with reference to the $D$. melanogaster genome.

\section{Comparison between Orthonome, OrthoDB and MSOAR2.}

We also compared the output of Orthonome to that of OrthoDB and MSOAR2 for 20 Drosophila species using the default parameters defined by Kriventseva, et al. [28] for OrthoDB and Shi, et al. [17] for MSOAR2.

\section{Results and discussion}

\section{Benchmarking against current pipelines}

To assess its performance, we applied Orthonome to datasets of 12 and 20 Drosophila species and compared our results to those generated with OrthoDB [29], Reciprocal bi-directional best hit [5], MetaPhors [11], OMA (groups and GETHOGS) [9] and MultiMSOAR2 [16, 17] using two measures described in Altenhoff, et al. [19]. Firstly, we calculated the average tree error (Robinson-Foulds distance between the reference species phylogeny and the 
tree obtained from orthologous clusters with all tested species [19]) for each method using the 12 and 20 genome sets. This test assumes that a true orthologous group with all species represented (1:1 ( $\mathrm{n}=$ all) orthogroups in the case of Orthonome) should give rise to the same phylogenetic tree as the overall species tree. Therefore a lower average tree error represents better predictive capacity of the pipeline. For the second of the measures we calculated the recall statistic, namely the number of complete $\left(1: 1_{(\mathrm{n}=\text { all })}\right.$ or maximal path search) orthologous group sets recovered by the pipeline, where a higher number indicates better resolution of orthologous relationships.

Application of the six pipelines above to the 12 high quality Flybase genomes showed Orthonome had the lowest average tree error rate and second highest recall (9538 1:1 (n=all) orthogroups, Fig. 2). By comparison, MultiMSOAR2, which had the highest recall (9595 1:1 ( $\mathrm{n}=$ all) orthogroups), also had the highest average tree error rate. The performance of Orthonome is likely to reflect improved pairwise orthologue identification due to use of the $S^{\prime}$ score. The latter uses the more accurate Smith Waterman alignment rather than heuristic BLAST alignments and accounts for weighted similarity per amino acid, which will further prefer the orthologous relationship between species over recently formed inparalogues. OMA (groups), which had the second lowest average tree error rate had nearly four-fold lower recall compared to Orthonome. Additionally, OrthoDB, which is the most widely accepted source of orthologues for the
Drosophila species [30], also had lower quality (3rd best) and recall (4th best) compared to Orthonome. Therefore, for the 12 genomes we found that Orthonome had the best combination of average tree error rate and recall compared to the six other pipelines tested.

Addition of the eight lower quality modENCODE genomes to the data set yielded a broadly similar pattern of differences among the pipelines [Fig. 2]. The recall rate for Orthonome was now rated 3rd best, but it still had the best combination of recall and accuracy. The lower number of 1:1 (n=all) orthogroups recovered by all pipelines from the 20 genome data set is most likely due to the lower quality annotations (e.g. chimeric and missing genes) in the eight modENCODE genomes [31].

\section{Detailed comparison of Orthonome with OrthoDB using the 12 genome data set}

The analysis of the 12 well curated Drosophila genomes in Flybase with Orthonome identified orthologous relationships for an average of $\sim 1.8 \%$ more genes in each species than did OrthoDB (13,516 vs 13,270), including resolving cryptic relationships between multiple genes and discovering more novel orthologous relationships. One such example is the orthologous pair: D. melanogaster gene FBgn0004554 and its D. sechellia counterpart FBgn0170274 (see e.g. in Fig. 3a/b and Additional file 1: Note 2). Orthonome also found $\sim 44 \%$ more $1: 1$ (n=all) orthogroups (9538 vs 6621) than did OrthoDB (Table 1, Additional file 1: Table S1 [29]). Specifically, it resolved

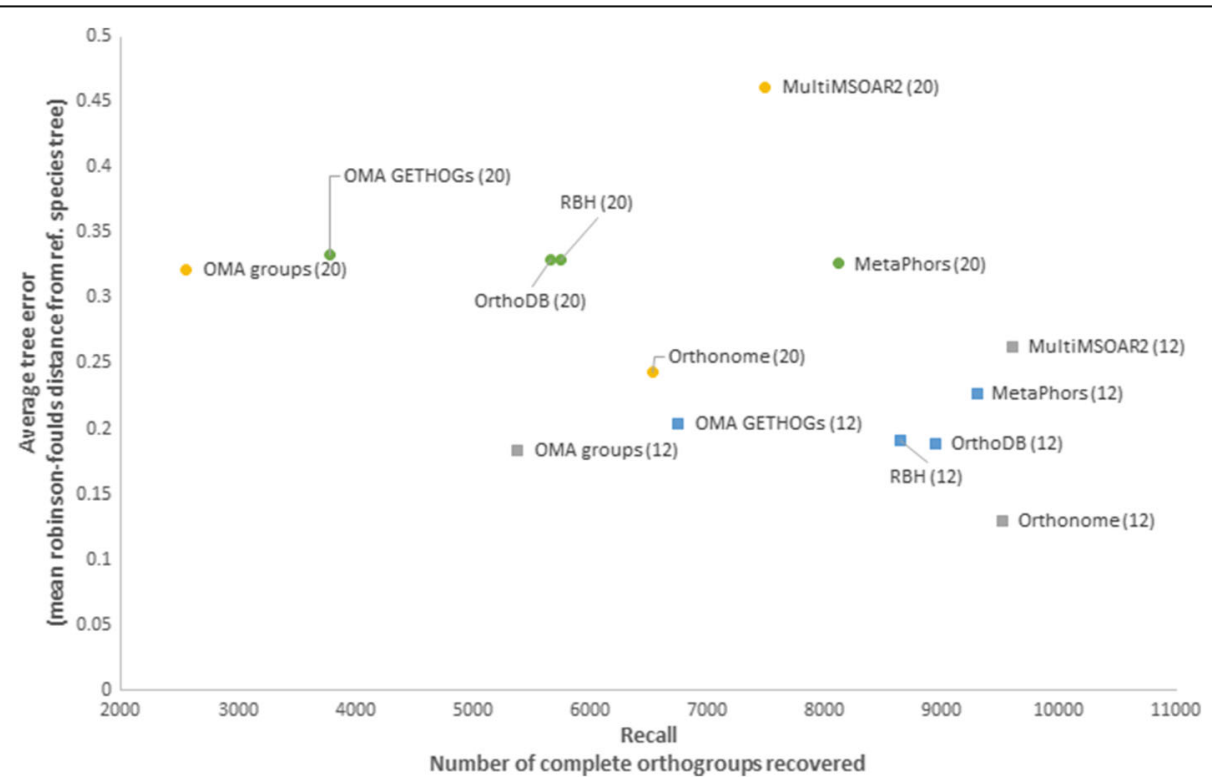

Fig. 2 Species tree discordance and orthologue recall tests for six pipelines on the 12 and 20 Drosophila species data sets. The circular markers represent the results for the 20 species data set while the square markers represent those for the 12 high quality Flybase genomes. The yellow circular markers and grey square markers represent pipelines that produce orthogonal orthologue sets with only one gene per species in a cluster. Orthonome provides a superior combination of low average tree error and high recall with both data sets 

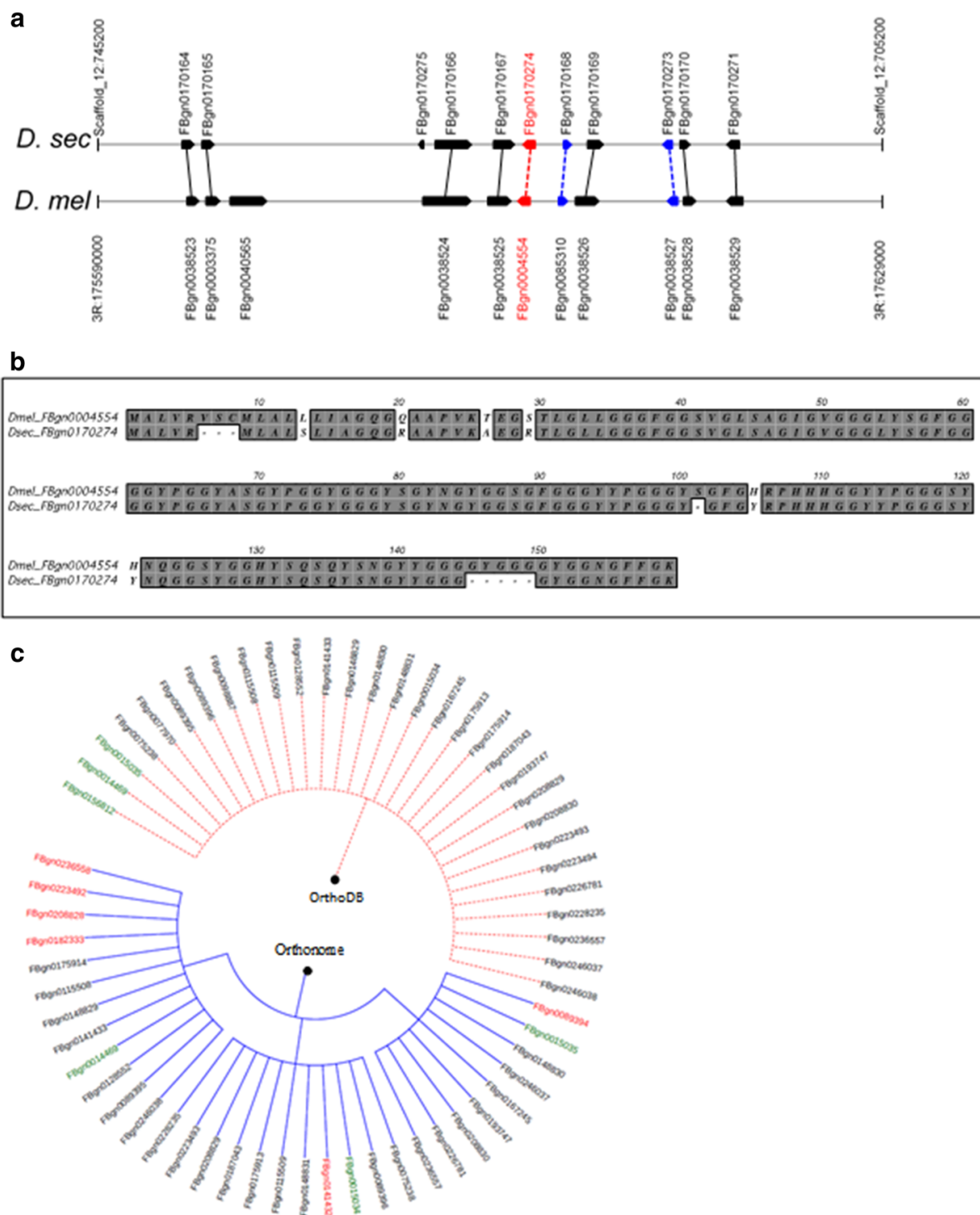

Fig. 3 Comparison between Orthonome, MSOAR and OrthoDB for orthologue identification across syntenically supported regions and fast evolving gene families. a A comparison of orthologue capture success in a $40 \mathrm{~Kb}$ syntenic region between D. sechellia and D. melanogaster. Orthologous relationships are indicated by vertical lines. Black lines = orthologous pairs supported by OrthoDB, MSOAR and Orthonome; blue lines = orthologous pairs detected only by MSOAR and Orthonome; red line = orthologous pair recovered only by Orthonome. $\mathbf{b}$ The newly recovered orthologous pair FBgn0004554 and FBgn0170274 in Panel A is supported by high ( $>90 \%)$ amino acid identity. c Orthonome is able to split OrthoDB orthogroup EOG7KHP47 (dotted orange clade) consisting of P450 monooxygenases into three independent orthogroups (solid blue clade). The three $D$. melanogaster genes are highlighted in green and the six additional genes that were allocated to the orthogroups by Orthonome only are marked in red. OrthoDB was unable to identify orthology to these six genes

an additional 2917 1:1 (n=all) orthogroups from among orthogroups that OrthoDB had left as 1:many or many:many sets (Fig. 3c and Additional file 1: Table S2). Furthermore, $94 \%$, or 6228 , of the $1: 1$ (n=all) orthogroups predicted by OrthoDB were also predicted by Orthonome.

The greatly enhanced capability of Orthonome to resolve 1:many and many:many into 1:1 relationships was evident in an analysis of 11 gene families associated with stress tolerances and prone to rapid amplification and loss in insects [32]. Orthonome could resolve 45-133\% more of these genes into 1:1 (n=all) orthogroups than OrthoDB (Additional file 1: Table S3). For example, cytochrome P450 monooxygenases (P450s) were classified into 51 1:1 (n=all) orthogroups by Orthonome compared to 22 by OrthoDB (Additional file 1: Table S3). An example of the improved orthologue assignment by Orthonome in a particular P450 gene cluster is illustrated in Fig. 3c. 
Table 1 Numbers of orthologues, orthogroups, inparalogues and gene births identified by Orthonome, OrthoDB, MultiMSOAR2, OMA groups and reciprocal best hit (RBH) using the same input data

\begin{tabular}{|c|c|c|c|c|c|c|}
\hline Comparison & Measures & Orthonome & OrthoDB & $\begin{array}{l}\text { Multi } \\
\text { MSOAR2 }\end{array}$ & OMA & $\mathrm{RBH}$ \\
\hline \multirow[t]{6}{*}{ Twelve FlyBase genomes } & Average number of genes per species & & & 15,446 & & \\
\hline & Average number of orthologues per species & 13,517 & 13,270 & 13,310 & 12,934 & 13,545 \\
\hline & Average number of inparalogues per species & 1519 & $N A$ & 1543 & NA & $N A$ \\
\hline & Average number of gene births per species & 400 & 2805 & 593 & $N A$ & $N A$ \\
\hline & Number of 1:1 (n=all) orthogroups & 9538 & 6621 & 9595 & 5380 & 8643 \\
\hline & Number of 1:1 (1<n<all) orthogroups & 6711 & 10,231 & 6042 & 15,493 & 9756 \\
\hline \multirow{6}{*}{$\begin{array}{l}\text { Twenty genomes (Twelve FlyBase } \\
+ \text { eight modENCODE) }\end{array}$} & Average number of genes per species & & & 15,055 & & \\
\hline & Average number of orthologues per species & 13,272 & 13,091 & 12,964 & 12,565 & 13,504 \\
\hline & Average number of inparalogues per species & 1305 & NA & 1422 & NA & NA \\
\hline & Average number of gene births per species & 468 & 2343 & 670 & $N A$ & $N A$ \\
\hline & Number of $1: 1$ ( $n=$ all) orthogroups & 6541 & 3912 & 7491 & 2555 & 5757 \\
\hline & Number of 1:1 (1<n<all) Orthogroups & 14,047 & 16,681 & 11,880 & 25,694 & 16,799 \\
\hline
\end{tabular}

Inparalogue predictions were carried out only in Orthonome and MultiMSOAR2. NA denotes the lack of inparalogue identification by OrthoDB and values that could not be calculated for MSOAR2 (since it has a different scoring method than Orthonome)

\section{Detailed comparison of Orthonome with OrthoDB using the 20 genome data set}

Compared to the 12 Flybase genomes above, the eight draft modENCODE genomes had significantly shorter post-assembly contig and scaffold N50 s (averages of 493Kb cf. $1.9 \mathrm{Mb}$ and $1.1 \mathrm{Mb}$ cf. $12.8 \mathrm{Mb}$ respectively; Additional file 1: Table S1) and more missing members of the universal insect gene set identified by BUSCO (0.06\% vs $1.65 \%$ respectively). Application of Orthonome to the eight draft modENCODE Drosophila genomes again achieved a further $0.5 \%$ improvement in orthologue recovery per species compared to OrthoDB. Compared to the 12 genomes analysis above, both pipelines had a reduction in the average number of orthologues recovery per species (OrthoDB: 1.8\%; Orthonome:1.3\%), which we can attribute to missing gene models in the additional draft genomes (Additional file 1: Fig. S1b). However, the number of 1:1 (n=all) orthogroups per genome obtained from the combined data sets using Orthonome was $67 \%$ higher than the number obtained with OrthoDB, bearing out the value of Orthonome even for poorer quality genome assemblies.

The ability of Orthonome to identify orthologues and in particular to resolve 1:many and many:many orthogroups into $1: 1$ (n=all) orthogroups with greater accuracy (Figs. 2 and 3c) also means that genes in each genome that do not fall into the latter class can be partitioned into three other classes, namely 1:1 (1<n<all) orthogroups, inparalogues and de novo gene births (Table 1). OrthoDB does not provide for such partitioning. As noted earlier, another pipeline, OMA, which also produces orthogonal 1:1 prediction, does so with very low recall while also having a higher discordance compared to species phylogeny (Fig. 2).
The numbers of genes assigned to the orthogroup, inparalogue and de novo gene birth categories will be less prone to error in the 12 genome analysis than the 20 genome analyses because the 12 genome analysis gives the best estimate of the number of $1: 1 \quad(\mathrm{n}=\mathrm{all})$ orthogroups, as explained above. As such, the finding of an average of 1519 inparalogues above and 400 de novo gene births per genome in the 12 genome analysis (Table 1) represents the best estimates yet available for these two crucial sources of genetic novelty in the genus. Nevertheless we observed a negative relationship between the number of 1:1 ( $\mathrm{n}=\mathrm{all}$ ) orthogroups per genome and the quality of genome assembly/annotation (see above, Additional file 1: Note 2). As a result, although they are the most up-to-date at the time of writing, the estimates of gene birth and inparalogues are still very likely to be overestimates of the true values for these quantities because of the residual errors in the underlying assemblies and annotations.

\section{Conclusion}

By comparing multiple pipelines we also find that Orthonome provides the best combination of accuracy and recall of orthologue assignments. Whereas previous pipelines are limited to classifying most genes in a genome into 1:many or many:many groups of orthologues, especially in draft genomes, Orthonome has separated the majority into 1:1 $(\mathrm{n}=\mathrm{all})$ orthogroups (representing evolutionary conservation) or inparalogues and gene births (representing the genetic novelty from which new functions may evolve). While the current study is limited to Drosophila species, the application of Orthonome in other species orders ([32] and unpublished data) demonstrates a broader usability of 
the pipeline, though it may be possible to further improve the quality of orthologue prediction using substitution matrices optimised for specific phylogenetic lineages. As the quality of genome assemblies and annotations continues to improve, Orthonome and any subsequent improvements on it should provide increasingly accurate estimates of the various sources of genomic variation fundamental to evolutionary change.

\section{Additional file}

Additional file 1: Supplementary notes, figures and tables. (DOCX 109 kb)

\begin{abstract}
Abbreviations
BL50: BLOSUM50 substitution matrix; BUSCO: Benchmarking Universal SingleCopy Orthologs; CSV: Comma separated values format; GFF3: General feature format version 3; HGT: Horizontal gene transfers; MCL: Markov cluster algorithm; OG: Orthogroup; OrthoXML: XML schema for storage of orthologue information; RSD: Reciprocal smallest distance; SOG: Super orthologue group; XLSX: Microsoft excel format
\end{abstract}

\section{Acknowledgements}

We thank Karl Gordon and Alexandre Fournier-Level for valuable discussion and suggestions. We also thank the NeCTAR cloud research facility for allocating significant computational resources for the continuance of the Orthonome database.

\section{Funding}

This work was funded by the Australian Science and Industry Endowment Fund (SIEF) and a NeCTAR computation grant to RVR. The funders had no role in study design, data collection and analysis, decision to publish or preparation of the manuscript.

\section{Availability of data and materials}

The source-code and raw data for the pipeline will be made available at https://bitbucket.org/rahulvrane/orthonome and the generated prediction database for Drosophila orthologues is available as a searchable website, www.orthonome.com

\section{Authors' contributions}

RVR and SFL conceptualised the study. RVR designed the pipeline and wrote the scripts. The web interface was conceptualised developed by RVR and databases implemented/maintained by TN. JO, AH and SFL helped interpret the biological significance of results. RVR, JO, AH and SFL wrote the manuscript. All authors read and approved the final manuscript.

\section{Ethics approval and consent to participate}

Not applicable

\section{Consent for publication}

Not applicable

\section{Competing interests}

The authors declare that they have no competing interests.

\section{Publisher's Note}

Springer Nature remains neutral with regard to jurisdictional claims in published maps and institutional affiliations.

\section{Author details}

${ }^{1}$ Bio21 Institute, School of Biosciences, The University of Melbourne, Melbourne, Victoria, Australia. ${ }^{2}$ CSIRO, Canberra, Australian Capital Territory, Australia. ${ }^{3}$ Department of Biological Sciences, Macquarie University, Sydney, New South Wales, Australia.
Received: 15 January 2017 Accepted: 21 August 2017

Published online: 31 August 2017

\section{References}

1. Gabaldon T, Koonin EV. Functional and evolutionary implications of gene orthology. Nat Rev Genet. 2013;14(5):360-6.

2. Vilella AJ, Severin J, Ureta-Vidal A, Heng L, Durbin R, Birney E. EnsemblCompara GeneTrees: complete, duplication-aware phylogenetic trees in vertebrates. Genome Res. 2009;19(2):327-35.

3. Mi H, Muruganujan A, Thomas PD. PANTHER in 2013: modeling the evolution of gene function, and other gene attributes, in the context of phylogenetic trees. Nucleic Acids Res. 2013;41(Database issue):D377-86.

4. Huerta-Cepas J, Bueno A, Dopazo J, Gabaldon T. PhylomeDB: a database for genome-wide collections of gene phylogenies. Nucleic Acids Res. 2008; 36(Database issue):D491-6.

5. Tatusov RL, Koonin EV, Lipman DJ. A genomic perspective on protein families. Science. 1997;278(5338):631-7.

6. Jensen $\sqcup$, Julien P, Kuhn M, von Mering C, Muller J, Doerks T, et al. eggNOG: automated construction and annotation of orthologous groups of genes. Nucleic Acids Res. 2008;36(Database issue):D250-4.

7. Schreiber F, Sonnhammer EL. Hieranoid: hierarchical orthology inference. J Mol Biol. 2013;425(11):2072-81.

8. O'Brien KP, Remm M, Sonnhammer EL. Inparanoid: a comprehensive database of eukaryotic orthologs. Nucleic Acids Res. 2005;33(Database issue):D476-80.

9. Altenhoff AM, Skunca N, Glover N, Train CM, Sueki A, Pilizota I, et al. The OMA orthology database in 2015: function predictions, better plant support, synteny view and other improvements. Nucleic Acids Res. 2015;43(Database issue):D240-9.

10. Linard B, Thompson JD, Poch O, Lecompte O. Ortholnspector: comprehensive orthology analysis and visual exploration. BMC Bioinformatics. 2011;12(1):11.

11. Pryszcz LP, Huerta-Cepas J, Gabaldon T. MetaPhOrs: orthology and paralogy predictions from multiple phylogenetic evidence using a consistency-based confidence score. Nucleic Acids Res. 2011;39(5):e32.

12. Dewey CN. Positional orthology: putting genomic evolutionary relationships into context. Brief Bioinform. 2011;12(5):401-12.

13. Dalquen DA, Altenhoff AM, Gonnet GH, Dessimoz C. The impact of gene duplication, insertion, deletion, lateral gene transfer and sequencing error on orthology inference: a simulation study. PLoS One. 2013;8(2):e56925.

14. Zhang M, Leong HW: BBH-LS: an algorithm for computing positional homologs using sequence and gene context similarity. BMC Syst Biol 2012, 6(Suppl 1).

15. Fu Z, Chen $X$, Vacic $V$, Nan $P$, Zhong $Y$, Jiang T. MSOAR: a high-throughput ortholog assignment system based on genome rearrangement. J Comput Biol. 2007:14(9):1160-75.

16. Shi G, Zhang L, Jiang T: MSOAR 2.0: Incorporating tandem duplications into ortholog assignment based on genome rearrangement. BMC Bioinformatics 2010, 11(1):10

17. Shi G, Peng MC, Jiang T: MultiMSOAR 2.0: an accurate tool to identify ortholog groups among multiple genomes. PLoS One 2011, 6(6):e20892.

18. Gogarten JP, Townsend JP. Horizontal gene transfer, genome innovation and evolution. Nat Rev Microbiol. 2005:3(9):679-87.

19. Altenhoff AM, Boeckmann B, Capella-Gutierrez S, Dalquen DA, DeLuca T, Forslund $\mathrm{K}$, et al. Standardized benchmarking in the quest for orthologs. Nat Methods. 2016;13(5):425-30.

20. Van Dongen SM. Graph clustering by flow simulation: Utrecht University; 2001.

21. Khan MA, Elias I, Sjolund E, Nylander K, Guimera RV, Schobesberger R, et al. Fastphylo: fast tools for phylogenetics. BMC Bioinformatics. 2013;14(1):334.

22. Katoh $\mathrm{K}$, Kuma $\mathrm{K}$, Toh $\mathrm{H}$, Miyata T. MAFFT version 5: improvement in accuracy of multiple sequence alignment. Nucleic Acids Res. 2005;33(2):511-8.

23. Suyama M, Torrents D, Bork P: PAL2NAL: robust conversion of protein sequence alignments into the corresponding codon alignments. Nucleic Acids Res 2006, 34(suppl-2):W609-W612.

24. Tange O. Gnu parallel-the command-line power tool. The USENIX Magazine. 2011;36(1):42-7.

25. NeCTAR Research Cloud [http://nectar.org.au/]

26. Jones P, Binns D, Chang HY, Fraser M, Li W, McAnulla C, et al. InterProScan 5: genome-scale protein function classification. Bioinformatics. 2014;30(9): $1236-40$. 
27. Marygold SJ, Leyland PC, Seal RL, Goodman JL, Thurmond J, Strelets VB, et al. FlyBase: improvements to the bibliography. Nucleic Acids Res. 2013; 41(Database issue):D751-7.

28. Kriventseva EV, Tegenfeldt F, Petty TJ, Waterhouse RM, Simao FA, Pozdnyakov IA, et al. OrthoDB v8: update of the hierarchical catalog of orthologs and the underlying free software. Nucleic Acids Res. 2015 43(Database issue):D250-6.

29. Waterhouse RM, Zdobnov EM, Tegenfeldt F, Li J, Kriventseva EV. OrthoDB: the hierarchical catalog of eukaryotic orthologs in 2011. Nucleic Acids Res. 2011;39(Database issue):D283-8.

30. Attrill H, Falls K, Goodman JL, Millburn GH, Antonazzo G, Rey AJ, et al. FlyBase: establishing a Gene Group resource for Drosophila melanogaster. Nucleic Acids Res 2015:gkv1046.

31. Tekaia F. Inferring orthologs: open questions and perspectives. Genomics Insights. 2016:9:17-28.

32. Rane RV, Walsh TK, Pearce SL, Jermiin LS, Gordon KH, Richards S, et al. Are feeding preferences and insecticide resistance associated with the size of detoxifying enzyme families in insect herbivores? Curr Opin Insect Sci. 2016; 13:70-6.

Submit your next manuscript to BioMed Central and we will help you at every step:

- We accept pre-submission inquiries

- Our selector tool helps you to find the most relevant journal

- We provide round the clock customer support

- Convenient online submission

- Thorough peer review

- Inclusion in PubMed and all major indexing services

- Maximum visibility for your research

Submit your manuscript at www.biomedcentral.com/submit
Biomed Central 